$\mathrm{ACI}$

$19,1 / 2$

82

Received 18 January 2019

Revised 3 March 2019

Accepted 4 May 2019

\section{Conceptual framework of a skill-based interactive employee engaging system: In the Context of Upskilling the present IT organization}

\author{
Siddarth Nair \\ Dublin Business School, Dublin, Ireland \\ Abhishek Kaushik \\ ADAPT Centre, School of Computing, Dublin City University, Dublin, Ireland, and \\ Harnaik Dhoot \\ Dublin Business School, Dublin, Ireland
}

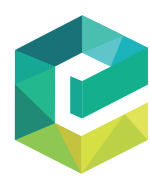

Applied Computing and Informatics Vol. 19 No. 1/2, 2023 pp. $82-107$ Emerald Publishing Limited e-ISSN: 2210-8327 p-ISSN: 2634-1964 DOI 10.1016/j.aci.2019.05.001

\begin{abstract}
Skills form an integral part of an organization for its efficient functioning. This research study investigates mechanisms to actively persuade employees and organizations to change their attitudes and perspectives towards skills through the design and implementation of a skill-based system. The aim of the project is to know if we can create a skill-focused climate in a software organization through a software system proposed by the researcher and if such a system will help in utilizing in-house employees for new opportunities rather than hiring new employees. Feedback was collected on the proposed skill-based system from software professionals and human resource managers through an online survey. Findings reveal that the proposed skill-based system will create a skill-focused climate in a software organization and also help in better utilization of internal employees for new skill requirements rather than directly hiring external employees.
\end{abstract}

Keywords Technical and soft skills, Employee engagement, Learning climate, Knowledge sharing,

IT organization

Paper type Original Article

\section{Introduction}

Employees with multiple skill sets are an asset to any organization. With the changing trends and advent of Artificial Intelligence, Machine Learning, Deep Learning, Blockchain technology etc. it is always better to have employees who are adaptable, flexible and open to embrace new

(C) Siddarth Nair, Abhishek Kaushik and Harnaik Dhoot. Published in Applied Computing and Informatics. Published by Emerald Publishing Limited. This article is published under the Creative Commons Attribution (CC BY 4.0) license. Anyone may reproduce, distribute, translate and create derivative works of this article (for both commercial and non-commercial purposes), subject to full attribution to the original publication and authors. The full terms of this license may be seen at http:// creativecommons.org/licences/by/4.0/legalcode.

Publishers note: The publisher wishes to inform readers that the article "Conceptual framework of a skillbased interactive employee engaging system: In the Context of Upskilling the present IT organization" was originally published by the previous publisher of Applied Computing and Informatics and the pagination of this article has been subsequently changed. There has been no change to the content of the article. This change was necessary for the journal to transition from the previous publisher to the new one. The publisher sincerely apologises for any inconvenience caused. To access and cite this article, please use Nair, S., Kaushik, A., Dhoot, H. (2020), "Conceptual framework of a skill-based interactive employee engaging system: In the Context of Upskilling the present IT organization", Applied Computing and Informatics, Vol. ahead-of-print No. ahead-of-print. https://10.1016/j.aci.2019.05.001. The original publication date for this paper was 07/05/2019. 
challenges. A software organization in particular, is an embodiment of professionals with technical and non-technical skills. The skills of the employees are used to solve complex real world problems, to come up with new products and services that touch the lives of people, helping them in one way or the other. On the other hand, the organization has a significant part in nurturing and encouraging their employees to develop their skills, which will not only help fulfill their aspirations but also help in better organization functioning with skillful employees. So, we can say skills play an important role for both employees as well as the organization.

\section{Literature work}

\subsection{Learning climate}

Eldor and Harpaz [1, p. 213] defines the learning climate as "the organizations beneficial activities in helping employees create, acquire, and transfer knowledge, has also been proposed as an antecedent of employee engagement." Organization learning climate fosters innovation [2]. Not only that, learning climate makes employees adaptable making them more equipped to face new challenges in the economy [3].

\subsection{Employee Up skilling}

Abdussalaam et al. [4, p. 96] states that, "Empirical evidence identifies that organizational success hinges on employees with the required knowledge, skills, and abilities and that employees effectiveness at learning new skills and knowledge is connected with the kind of learning technique the organization adopts." Here, it clearly says that the organization"s learning system plays a crucial role in imparting effective knowledge and skills to the employees. The skills-based system should be intuitive and encouraging enough to make the employees as well as the employers worth to use it.

\subsection{Knowledge management}

Abhishek et al. [5] describes in detail the importance of knowledge in an organization in the customer relationship management scenario and how customer knowledge can lead to Customer Intelligence. Similarly, in the context of enterprise software organization, deriving insights and intelligence from the skills data obtained from the employees can yield business value adds to the organization. Also, considering an enterprise system focusing on skills will have to deal with skill documents like skill certificates and related resources, effective content management becomes a necessity. Sudhanshu et al. [6] compares various content management systems which could be used for the principal investigation of our IT based system that can provide us in-depth knowledge of the process of managing the content in the organization. That can enhance our vision and prospective for the skill-based system. These studies [5,6] lays out a huge potential for the organizations to use the skill-based system for better awareness among the employees and provide them an opportunity to update their technical and non-technical skills.

\subsection{Knowledge sharing}

Ozer and Vogel et al. [7, p. 134] states, "Receiving knowledge from other software developers in the company is positively related to the performance of the knowledge-receiving software developers." Knowledge sharing leads to innovation [8] and helps in employees' job satisfaction [9]. So, having a skills focused system that encourages employees to demonstrate their learning to other employees will not only help in their upskilling but also create a knowledge sharing organization culture that will encourage employees to share their learning.

\subsection{Employee engagement}

Felstead et al. [10, p. 1667] clearly mentions, "employee involvement is likely to play a significant role in the process of upskilling the workforce". So, the organization must make

Framework of employee engaging system 
$\mathrm{ACI}$

$19,1 / 2$

84

measures that the skills-based system has enough features to keep the employees involved. The skills-based system should encourage employees self-directedness and Lejeune et al. [11] clearly says that such employees" self-directedness helps in their personal development, thereby boosting their workplace performance.

\subsection{Gamification}

Deterding et al. [12] defines gamification as "the use of game design elements in non-game contexts". DuVernet, Amy and Popp [13] describes in detail how gamification elements like points and badges helps in better user engagement and motivation. So, having a skill-based system that uses these gamification elements will help to create an encouraging and intuitive experience for the employees.

\section{Problem statement and significance}

Currently there are systems that use gamification for employee engagement, but they do not focus on upskilling employees. There are systems that allow employees to enter/update their skills, but they are not used as a tool to create a skills-focused culture within an organization. Appraisals in organization focus on project level metrics and maybe some personal development objectives, but skills are not used as a driving factor. From an organization standpoint, in case of skills deficit, mostly they recruit new employees with therequired skillset and experiencerather than upskilling existing employees who have bandwidth and are interested to acquire new skills. Currently, organizations does not provide a common and encouraging platform for employees to showcase their skill sets. Also, employees find it difficult sometimes to understand the required level of skill set for a given role and how to achieve them in a clear manner. So, it is worth researching if we can figure out a way to come up with a system that focuses on employee skills as an active medium.

\subsection{Motivation and goal}

The aim of the project is to know whether a change in perspective can be brought in a software organization focusing on upskilling employees in an intuitive and an encouraging manner, through the use of a skill-focused software platform.

\subsection{Objectives of the project}

Following are the objectives of this study:

1. To know whether a skill-based software system can help encourage employees to proactively learn and share knowledge in an organization creating a vibrant, dynamic and enthusiastic leaning climate in a software organization.

2. To know whether a skill-based system will serve as a reliable medium to reach out to the right expert to resolve technical queries.

3. To know whether a skill-based software system will help in effective learning of emerging trends like artificial intelligence, machine learning etc.

4. To know whether a skill-based system can prevent recruiting new employees in case of skill deficit.

\section{Research questions}

The research questions are inclined towards the examination of the potential of skill-based system which can enhance the present IT companies scenarios and can be a milestone not only by upskilling the resource in the organization but also saving the extra revenue on external training. The whole study is motivated to finding the answer of the following research questions. 
4.1 How can you create a skills focused climate in a software organization through a software system?

Imagine a system which showcases the skills acquired by the employees, an ability to view other employees' skills to know who is good at what, different ways to learn/improve their skills in an intuitive manner and a functionality that helps to connect to other employees with required skill set for any query.

This system will help to create a skill focused learning climate within an organization which will provide an opportunity for employees to learn and improve.

\subsection{Will a skill-based system help in utilizing in-house employees for new opportunities rather}

than hiring new employees?

In case of skill deficit, employers prefer to hire new employees with required skill set and experience rather than upskilling existing employees. If an organization has huge bench strength (i.e. employees who are not allocated/billed against project), they can use this system to identify whether any of these employees can be provided an opportunity. For example, consider there are 50 employees with DOT NET technology as their core skill and they are not allocated to any project and waiting for an immediate opportunity. But, out of these 50 employees, 3 employees have updated 'Python' programming language as a secondary skill. Now, if there comes a new requirement for 'Python' developers, these 3 employees can be provided an opportunity rather than hiring new employees which would eventually help in saving costs, time and efforts.

\section{Methodology}

As shown in Figure 1, following steps were taken:

1. Identify the minimum viable features to incorporate in the software system Münch et al. [14, p. 1] defines a Minimum Viable Product (MVP) as "an artefact that may be incomplete in functionality or quality, but displays characteristics that

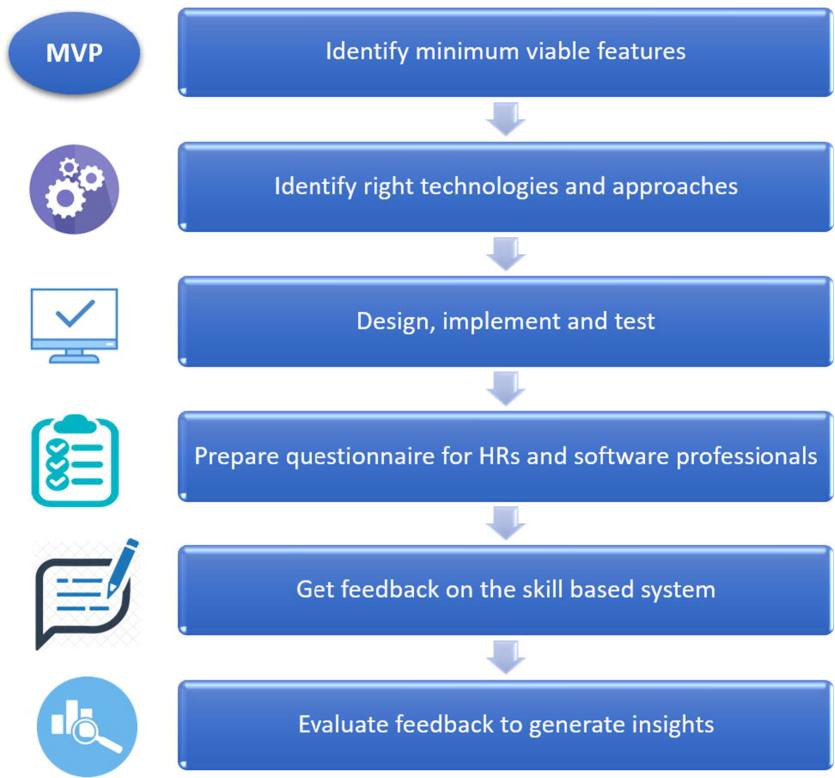

Framework of employee engaging system 
$\mathrm{ACI}$

$19,1 / 2$

86

allows determining its customer value". The researcher selected the features to implement that will best depict the product vision of a system that will create a skillfocused climate. These features were subjected to modifications while development to come up with a simple skill-based system.

\section{Identify the right technologies and approaches to implement the features as a web application}

Table 1 lists the development tools and technologies used in the project. Highcharts is used for showing charts in dashboard. Log4Net is used for logging system exceptions to a text file under Logs folder in the project folder. jQuery Easy Ticker is used for sliding notifications and Animate.css is used to animate onscreen elements for better user experience.

\section{Design, implement and test the application}

This phase involved the following aspects of implementation:

(a) Database design - Using SQL Server as the development environment, a database was created along with tables, schema, stored procedures and functions. These database objects were used to interact with the front-end of the system.

(b) UI design - Marvel prototyping tool was used to identify the minimal viable features to be considered for development. Then after, actual screens were developed using ASP.NET MVC framework which interacts with the back-end system to read and update data.

(c) Web API Design - This layer acts as an interface between the front-end and the back-end system forming a layered architectural design and abiding separation of concerns principle.

(d) Logic implementation - This phase corresponds to the business logic aspect of the development of the system. All the business rules and constraints defined under the artefact workflows were implemented.

(e) Unit testing - This phase of development focuses on testing the coded components of the system with respect to its expected behavior. The bugs identified were fixed.

(f) System testing - Here, in this stage of development, after the individual components were tested separately, post-integration, the whole system was tested

Technologies

Development Environment(s)

Prototyping tool

Configuration Management

tool

Project Management tool

Third Party Libraries

${ }^{1}$ https://marvelapp.com/.

2 https://github.com/.

3 https://trello.com/.

${ }^{4}$ https://www.highcharts.com/.

5 https://www.nuget.org/packages/log4net/.

${ }^{6} \mathrm{https} / /$ plugins.jquery.com/easyticker/.

${ }^{7} \mathrm{https}: / /$ daneden.github.io/animate.css/.
ASP.NET MVC, Web API, C\#, SQL Server, HTML5, CSS3, Bootstrap,

Javascript, jQuery

Visual Studio 2017, Microsoft SQL Server Management Studio 2017

Marvel $^{1}$

GitHub $^{2}$

Trello $^{3}$

Highcharts ${ }^{4}$, Log $4 \mathrm{Net}^{5}$, jQuery Easy Ticker ${ }^{6}$, Animate.css ${ }^{7}$
Table 1.

Development technologies and tools. 
to identify any bugs or unexpected behavior. All the bugs identified were fixed and the system is made stable.

The researcher has used Agile software development approach of incremental development using the framework of Scrum. The artefact developed as a part of the research is a prototype. As per Sommerville [15, p. 62], "A prototype is an early version of a software system that is used to demonstrate concepts, try out design options and find out more about the problem and possible solutions".

\section{Prepare a questionnaire to obtain feedback}

The target population includes software professionals and human resource managers working in a software organization. So, two questionnaires were developed with relevant set of questions. The questionnaires were prepared using Google Forms ${ }^{1}$. The questionnaires include a combination of both open as well as closed questions. The questionnaires mainly focused on extracting feedback about the proposed skill-based system with regards to its ability to create a skill-focused climate in the organization and whether this system will help in better utilization of in-house employees for new skill requirements. At a high level, each questionnaire is divided into three sections as described below:

(a) General - This section includes a set of questions that inquires on the following aspects:

i. Level of experience - The researcher believes participant work experience in the software industry is an important aspect to consider while drawing inferences after the data is collected. The researcher believes the responses from the higher experienced bracket will yield more value to the research.

ii. Existing skill-based system - Here, the questions ask for existing skill-based systems in the participant's organization and also if they are aware of the technical experts in their organization to reach out in case of technical queries.

iii. User specific questions - For software professionals, the questionnaire intends to know about their inclination towards skills. For human resource managers questionnaire, their current recruitment approach in case of skills deficit is inquired.

(b) Skill-based system - The researcher has prepared a short research video presenting the need for a skill-based system, the features of the system and the benefits, if used in a software organization at a high level. The video was uploaded to YouTube ${ }^{2}$ and the URL was embedded in this section to inform the participants on the need of the proposed skill-based system, its features as well as benefits. The researcher believes presenting such a video about the system will help the survey participants to answer the feedback questions in the following section.

(c) System feedback - This section includes questions to understand the participants level of agreement with regards to their perceptions about the proposed skill-based system, foreseeing the benefits at an individual level as well as for the organization to create a skill-focused climate. Also, this section includes three open-ended questions; couple of those aligned to the research questions requesting their reasoning on their perception how the system will affect the skill climate in their organization and utilization of in-house employees for new skill requirements. The third open question is to get any other comments or suggestions the participants wish to share. As rating questions are a good way

Framework of employee engaging system 
$\mathrm{ACI}$

$19,1 / 2$

88

to obtain participants' opinion [16], a Likert-scale rating scale of 5-point from Strongly agree to Strongly Disagree is used to collect participants' feedback on the skill-based system.

\section{Reach out to HR, managers and software developers to obtain feedback}

The subjects of the questionnaire will be Human Resource Managers, project managers and software developers. The targeted sample size will be roughly about 10-20 people based on availability, of which $50 \%$ will be managers.

\section{Evaluate the feedback to determine whether such a system will help to build a skills-focused culture in a software organization}

Once the feedback was obtained, the responses were analyzed to find out if such a skill-based system has a potential to meet the stated aims and objectives. The Findings \& Discussion section describes in detail the feedback received from the survey participants.

\section{Artefact}

\subsection{System overview}

As the idea is to see if a skill-focused platform can help improve employee engagement through a software system, to prove the possibility practically, a software system is required. The three main actors in the proposed skills-based system are employees, managers and skill experts as shown in Figure 2.

The employee initially will enter his/her skills into the system. Based on the entered skills, the system will then ask more questions to the lowest possible level sub-question, finally presenting the employee with a skills dashboard. Figure 3 illustrates the high level conceptual design diagram of the skill-based system. The employee skills dashboard will help with the following information:

- Required role level skills

- Points acquired for each entered skill

- View other employees" skills

- Ways to improve the existing skills as well as learn new skills via certifications, demonstrations using a proof-of-concept, learning by attending a training/ demonstration or resolving technical queries.

\section{SKILL BASED SYSTEM}

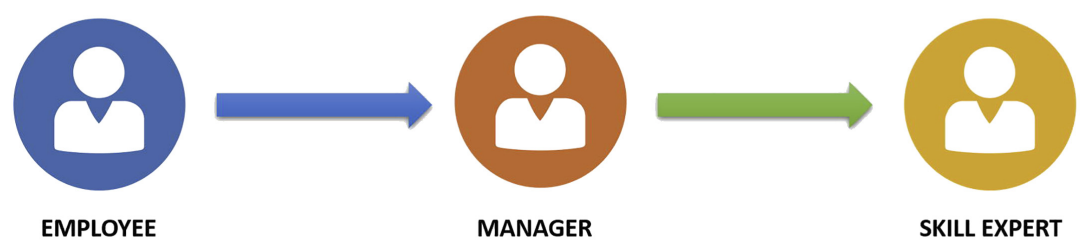

Figure 2.

System Actors.
- Enter his skills

- Improve his skills

- View others' skills

- Ask/Clarify Queries
- Provide required skills and points in the system for their project(s)

- Approve/Dis-approve employee skill request
- Prepares drill down questions for a skill and set points for sub-skills

- Provide feedback points after skill demonstration 


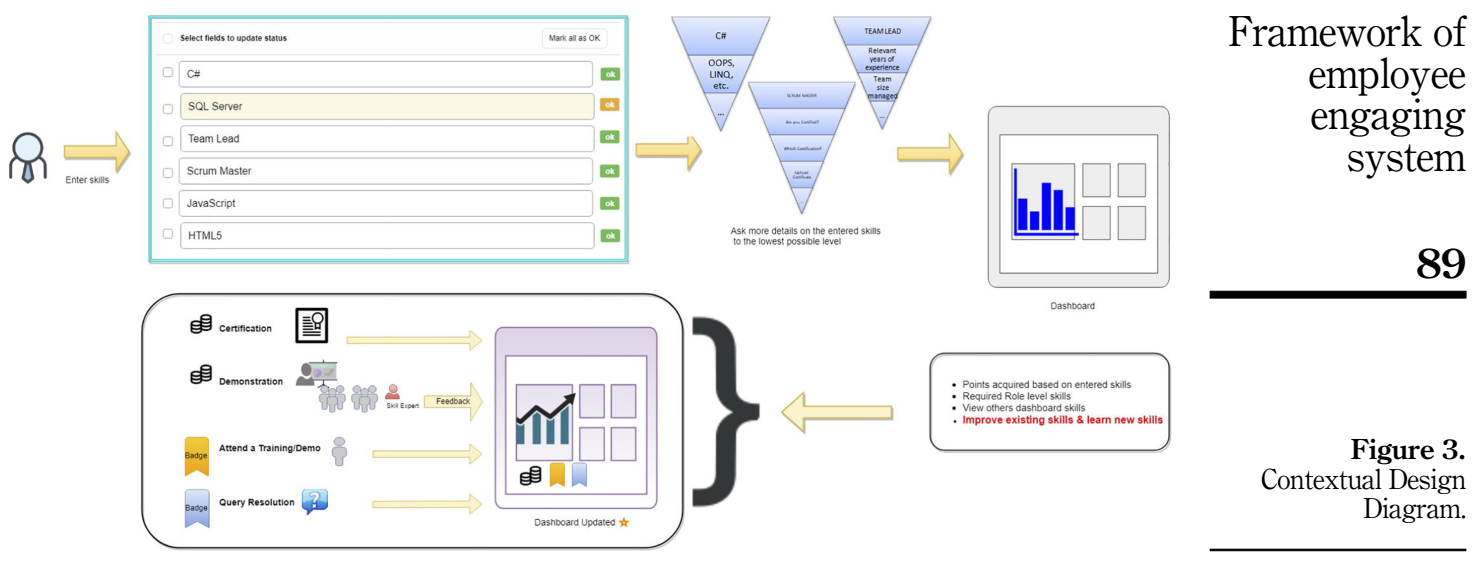

The system will follow points and badges system. Skill 'points' will be allocated to the employees who do certification and demonstration using a proof-of-concept whereas, 'badges' will be assigned to those who attend demonstrations/training and resolve queries. There will be a skill expert present at all times to validate the employee's knowledge of the new skill and will provide feedback as well as update skill points in the system which will then be reflected in the employee's dashboard. Also the skill expert needs to feed into the system, appropriate drill down questions for the respective skills.

Managers have the responsibility to feed into the system required skill points for their projects. Also they can approve/reject their sub-ordinates' requests in the system.

\subsection{Workflows}

At a high level, there are five major workflows in the skill-based system. Each of these are explained in detail below:

6.2.1 Employee dashboard creation. Figure 4 shows the dashboard creation activity diagram for an employee at a high level. One of the important and distinctive feature of the system is the drill down questions for each of the entered skill by the employee. If an employee enter a skill initially to the system, he/she will be confronted with an associated set of questions based on that skill to further refine the high level skill to more specific subject areas within that skill. This is owing to the fact that an employee who claims to know a high level skill may not know every subject modules within that skill area. So it is better the system tries to identify and separate what he/she actually knows and doesn't know which will lead to better focus for improvement. Consider the following examples:

1. If an employee enters ' $\mathrm{C \#}$ ' as his/her skill, the next drill down question can be to select the knowledge and expertise in Classes, Structures, LINQ, Inheritance, Polymorphism etc. The employee may know only 'Classes' out of these. This increases the reliability of the system in case of query resolution. Now, if someone in future wants to approach for a query regarding C\# - LINQ problem, this employee even though he knows C\# at a high level won't be considered in the list of relevant candidates to approach.

2. If an employee enters 'Scrum' as his skill, the first drill down question can be 'Are you certified?'. If the employee answers 'Yes', the next drill-down question can be 'Are you certified from Scrum Alliance or Scrum.org or other?'. And based on the answer, the next question can be to upload certificate as a proof and so on. 


\section{$\mathrm{ACI}$ \\ $19,1 / 2$}

90

Figure 4.

Activity Diagram: Employee Dashboard Creation.

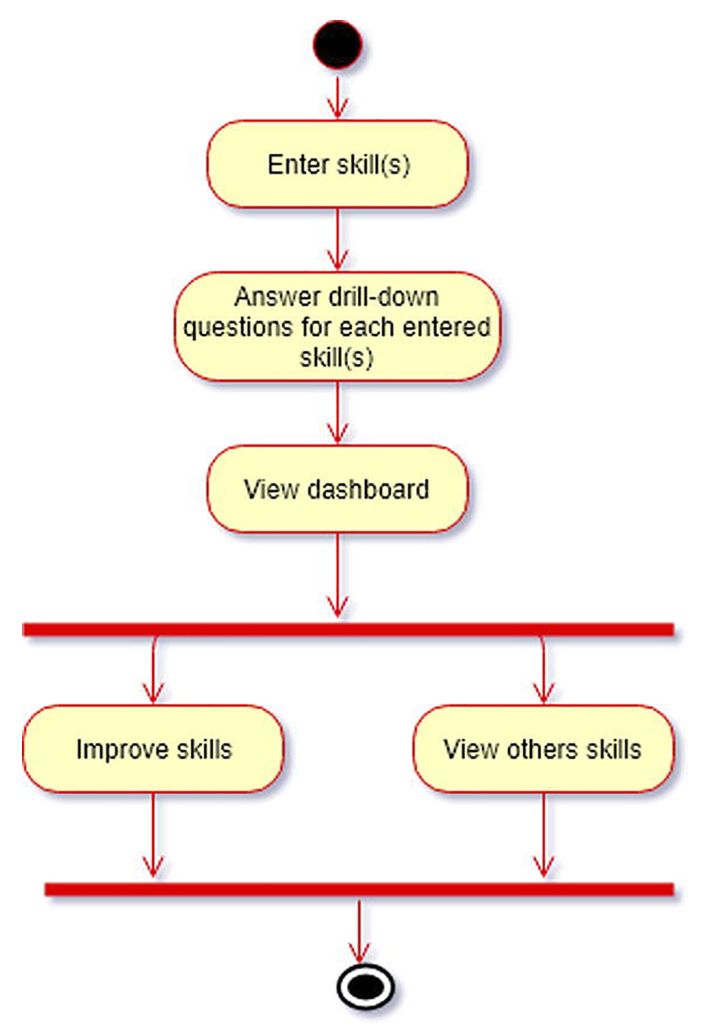

A point to note here is that these drill down questions like mentioned above is to be prepared and uploaded to the system by the respective skill experts. A C\# skill expert will prepare and upload drill down questions for C\# skill. Similarly, for other skill sets the respective skill expert will feed in the drill down question set to the skill system. Once all the drill down questions are answered, the employee will be presented a dashboard page which will display visual graphs to indicate all the skills and associated skill points. Each skill will have a range of $0-100$ skill points. The dashboard will show the required skill points for the employee for his role and the project so that he or she can focus on ways to meet the lacking skill points.

6.2.2 Improve skills by certification. Figure 5 describes the activity diagram for how the employee can improve his/her skills via certification. Here the skill expert verifies and validates the uploaded certificate on two aspects:

1. The certificate is authorized from the organization policy standpoint. In other words, the organization may consider only certain certification bodies as authorized or trusted.

2. The uploaded certificate for a skill can be considered for the entire 100 skill points for that skill or lesser. All of these will be defined by the skill-expert in compliance with the organization policies.

Once the uploaded certificate has been validated and approved by the skill expert, the associated skill points will be reflected in the employee skill dashboard. 


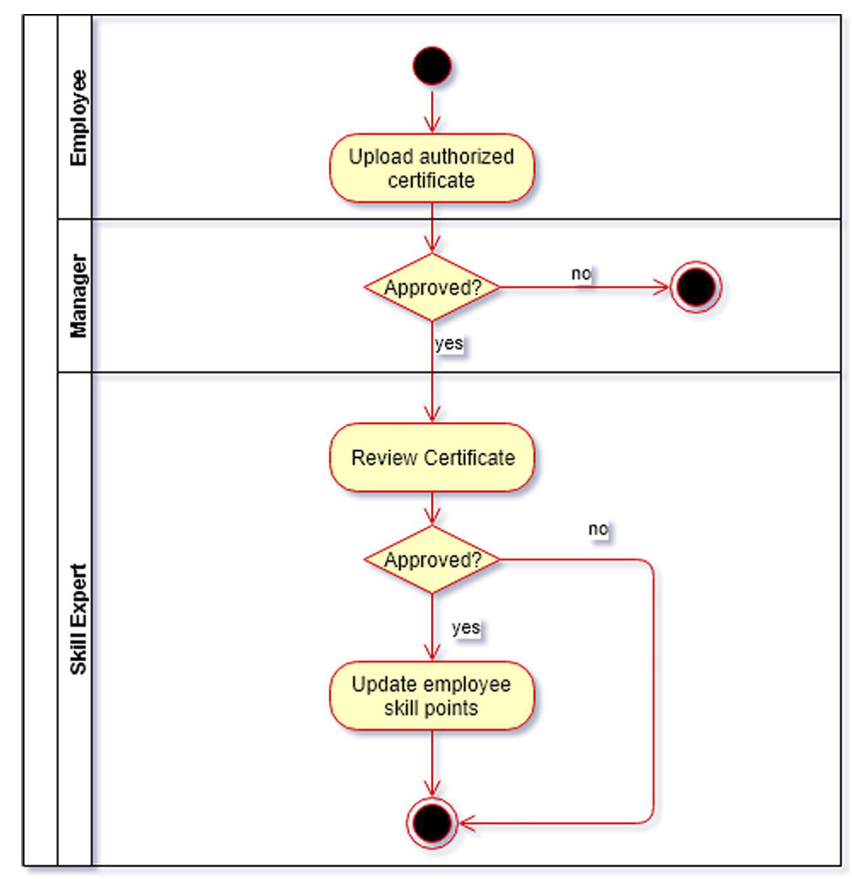

Framework of employee engaging system

91

Figure 5.

Activity Diagram: Improve skills by Certification.

6.2.3 Improve skills by demonstration using a proof-of-concept. Figure 6 shows the activity diagram of this work flow. This method involves the presence and feedback of the skill expert for evaluating the targeted skill by the employee. The system will have a functionality to 'Create a Demo event'. The created demo event will be displayed as a notification on every employees' dashboard with an option to select if they are interested or not. The demonstration will be conducted only in the presence of a skill expert. The employee's skill will be validated by the skill expert and if satisfactory, appropriate skill points will be updated in the employee's dashboard. One of the important feedback point can be effective communication. This is an important feature of this skill-based system because this channel focuses on knowledge sharing, leading to creativity and innovation. This knowledge sharing culture will help towards effective communication and the use of a proof-of-concept will bring in practical thinking and application of learning.

6.2.4 Improve skills by attending demonstration/training. Figure 7 depicts the activity flow for improving the skills by attending a demonstration. The above two methods will help earn skill points to the employees but this method will help earn skill 'badges'. Although skill badge is not in par with skill points, earning multiple and diverse skill badges will encourage employees to help others in need.

6.2.5 Resolving queries. Figure 8 shows the high level work flow steps. This is a channel to connect to the right person to resolve employees' queries or to seek guidance. The drill down questions helps in better reliability of the filtered candidates to approach for query resolution. This platform will help to identify people who are better fit to resolve the query based on skills. For a query related to skill X, on search the system may show lots of employees who have that skill knowledge, but those can be sorted, in terms of experience and availability to connect to the best of the lot.

Here, once the query is resolved, the resolving employee will be assigned a skill badge as a reward or appreciation. An employee can accumulate different skill badges which will be 


\section{$\mathrm{ACI}$ $19,1 / 2$}

\section{2}

Figure 6.

Activity Diagram: Improve skills by demonstration.

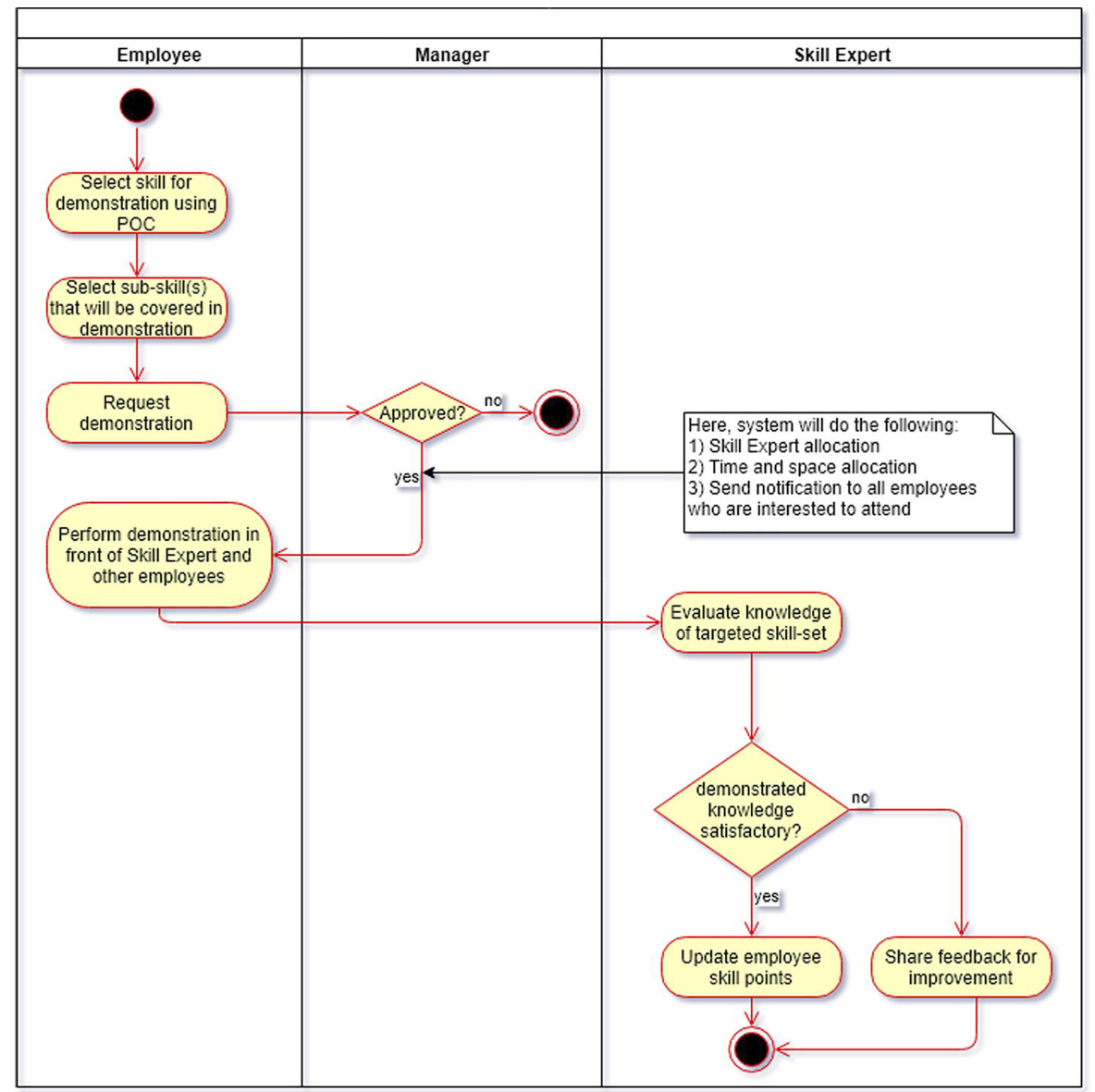

shown in the employee skill dashboard. This will inculcate encouragement and proactiveness within employees to earn as many badges as possible.

In a general sense, all these above workflows goes through manager approval and validation of each skill improvement method involves the approval of the respective skill expert for the concerned skill. Please note, this is just a basic safeguard added to this system. Additional and more sophisticated safeguards can be added on top of this system to ensure proper utilization of the system that suits the organization.

\subsection{Benefits}

1. Reliable system for query resolution: Rather than showing high level skills which many of the enterprise systems does, this system will store skills drilled down to the lowest possible level making it more reliable for query resolution.

2. Encouraging work-life balance: This system can be used to promote Corporate Social Responsibility (CSR) and Hobbies within employees to encourage work-life 


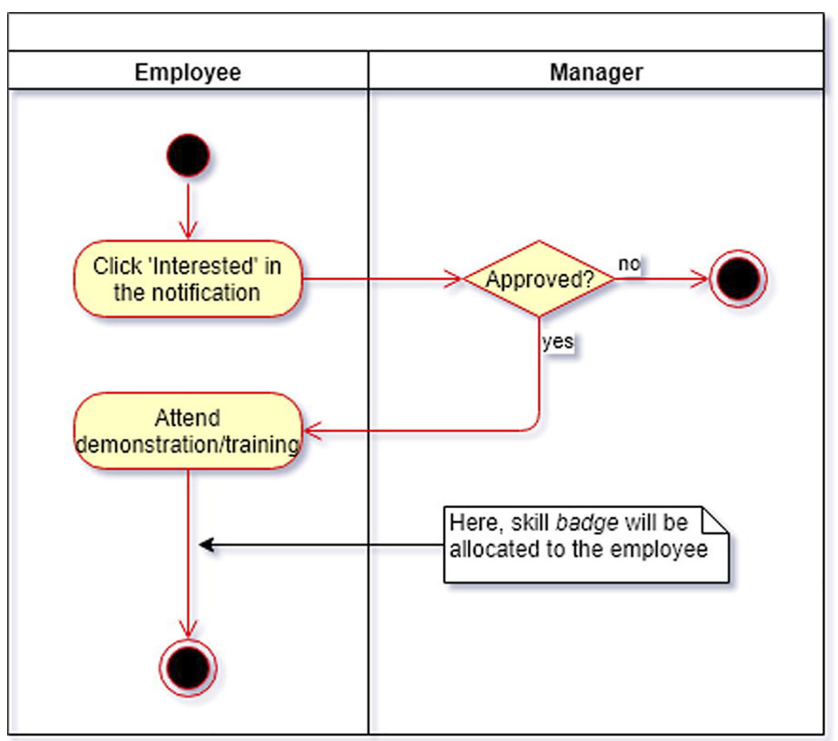

Framework of employee engaging system

balance. For example, a mandate can be put across through this skill-based system that employees have to gather at least 10 skill points per performance cycle by taking part in hobbies and extra-curricular activities in the organization.

3. Platform for new project requirements: This system can also be used for attracting free employees to new projects with required skills on the basis of skill points. Say, the organization is in need for a resource with some background in Python programming language. Here, a notification can be pushed through this system something like 'New project starting MM/DD/YYYY - Skills required: PYTHON with minimum 20 skill points'. This notification will be shown to all employees in the organization. The employees who are not allocated to any project or in bench and interested to get that opportunity can go for it. If the employee has already accumulated those required skill points, he/she can apply right away for this opportunity. For employees who doesn't have the required skill points and interested to grab that opportunity to work in that project can collect the skill points either by learning and uploading a valid certificate of Python or conducting a live demonstration in front of the skill expert to prove his/her knowledge and avail required skill points.

4. Platform for hackathon/programming contest: This system can be utilized to attract right resources for a programming contest. For example, a notification can be put across through the skill system like below: 'Team Programming contest: Skills required - C\# 25 points, SQL 50 points, HTML5 35 points'. The employees who satisfy either of the skill points criteria can apply to be a part of the contest.

\subsection{Application dashboard}

Figure 9 shows the dashboard of the proof-of-concept skill-based system. As per the dashboard, the employee has met the required skill points for Java and require just 25 


\section{ACI \\ $19,1 / 2$}

\section{4}

Figure 8.

Activity Diagram: Resolving queries.

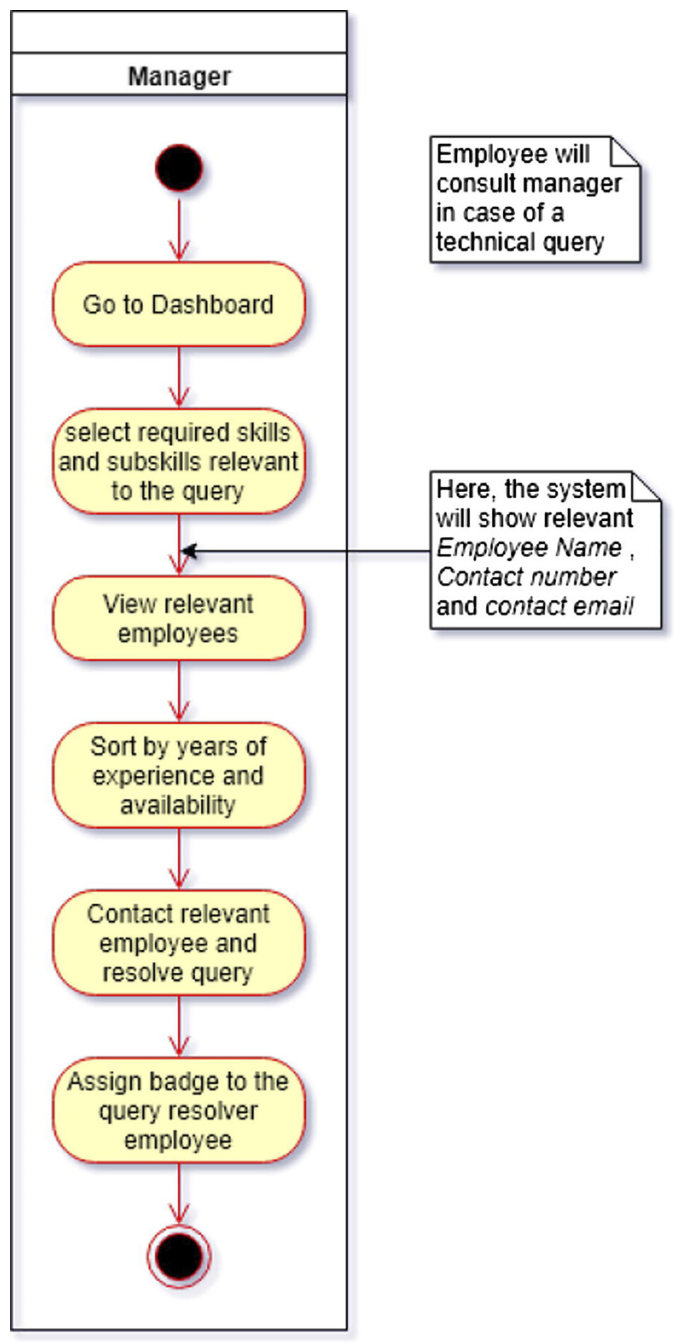

points for C\#. Also, skills Pyt h on and SQL are required with 50 skill points each, but the employee hasn't acquired any at the moment. The employee has earned 4 badges after attending demonstrations and 1 query badge after helping someone with their query. The notifications section to the bottom of the screen shows the upcoming demonstrations. Please refer Appendix for the screenshots of the proof-of-concept web application.

\section{Findings}

The questionnaire was distributed online to 50 software professionals and 30 human resource managers. Out of 50 software professionals, 48 participated in the survey, whereas out of 30 human resource managers, only 11 participated in the survey. So the response rate was $96 \%$ from software professionals, whereas only 37\% from human resource managers. For responses to the software professional questionnaire, the researcher contacted software 


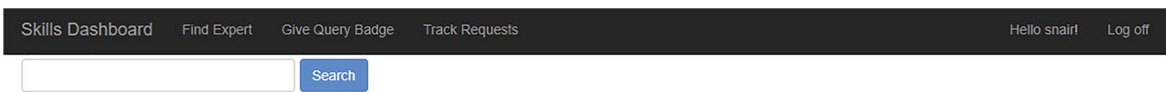

\section{Dashboard}
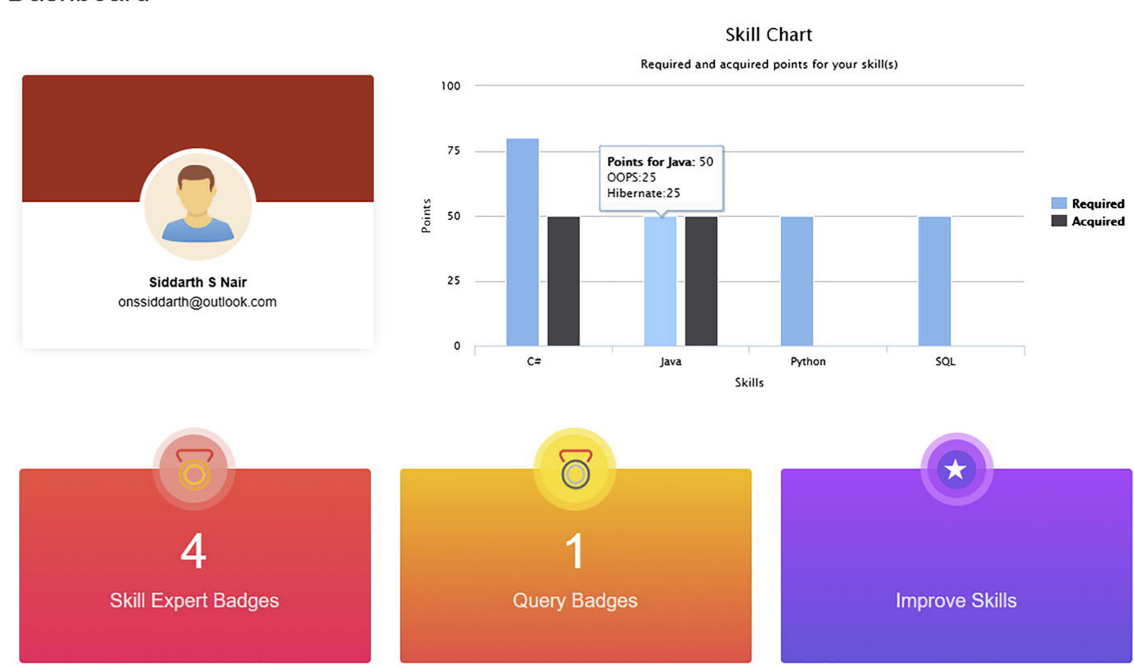

Framework of employee engaging system

95

\footnotetext{
\begin{tabular}{|l|}
\hline \multicolumn{1}{|c|}{ Event Notifications } \\
\hline Skill: SQL (DDL) \\
Demo on DDL (SQL) by Siddarth Nair on 09-01-2019 \\
10:00:00 at AS2.3 \\
\hline
\end{tabular}
}

Figure 9. Skill Dashboard.

developers, senior software developers, technology architects, project managers and delivery managers working in software organizations across Ireland, United Kingdom, Germany, India and United States of America. For responses to the human resource manager questionnaire, the researcher approached human resource managers working in software organizations across Ireland, United Kingdom and India.

\subsection{General perspectives}

$92 \%$ of software professionals who participated in the survey are from large software organizations. As far as human resource managers who participated in the survey are concerned, almost $55 \%$ of them are from large software organizations, around $27 \%$ from small organizations and around $18 \%$ from medium sized organizations. Almost $46 \%$ of the human resource managers and around $31 \%$ of the software professionals have answered their organizations doesn't have any skill focused system. $80 \%$ of the software professionals who said they don't have any existing skill-based systems in their organization are from large organizations and $20 \%$ from small organizations. Around $33 \%$ of the software professionals and almost $64 \%$ of the human resource managers say their organization focuses on upskilling employees through knowledge sharing by demonstration of skills. Almost $42 \%$ of the software professionals and around $45 \%$ of the human resource managers are not aware of the technical experts of various domains in their software organization. Almost $64 \%$ of the human resource managers say that they directly recruit new employees with the required skill(s). Around $37 \%$ of the software professionals are not aware of the required skills for their 
ACI

$19,1 / 2$

96

role. Majority of the software professionals and the human resource managers liked the feature of knowledge sharing through demonstration of skills more than the rest.

\subsection{Ability of the skill-based system to create a skill-focused climate}

As shown in Figure 10, more than $90 \%$ of the software professionals as wellas theHuman resource managers whoparticipated in thesurvey thinks this proposed skill-based system willcreatea skillfocused learning climate in the software organization. The cross tabulation as shown in Figure 11 shows the distribution of participant responses with respect to their experiences. Almost $94 \%$ of thesoftwareprofessionals believe this system will createa skill-focused climate in theorganization and the remaining $6 \%$ are not sure. Out of the total respondents who answered 'Yes', almost $38 \%$ comes from the experience range of $0-4$ years, almost $27 \%$ comes from 6 to 10 years of experience, $20 \%$ comes from 4 to 6 years of experience and the remaining almost $16 \%$ comes from over 10 years of experience. All the software professionals who falls within the experience bracket of $0-4$ years and over 10 years have responded 'Yes'. Almost $86 \%$ of the respondents who fall in the experience range of $6-10$ years have answered 'Yes' and the remaining $14 \%$ are not sure. As far as respondents in the $4-6$ years of experience are concerned, $90 \%$ of them have answered 'Yes' and theremaining $10 \%$ are not sure. Almost $91 \%$ of the human resource managers believe this system will create a skill-focused climate in the organization and the remaining $9 \%$ are not sure. Out of the totalrespondents whoanswered 'Yes', $40 \%$ each comes from theexperiencerange of $0-4$ yearsand $6-10$ years and the remaining $20 \%$ comes from 4 to 6 years of experience. All the human resource managers who falls within the experience bracket of $0-4$ years and 6-10 years have responded 'Yes'. $80 \%$ of the respondents who fall in the experience range of $6-10$ years have answered 'Yes' and the remaining $20 \%$ are not sure.

Figure 12 describes the thematic analysis done by the researcher on the open-ended question, to identify from the participant responses, the factors in the skill-based system that contributed towards creating a skill-focused climate. The responses to the open-ended question were run through thematic analysis to identify common themes and computing the basic statistics of frequency and proportion. Table 2 highlights the common themes identified by the researcher in reference to the participant's answer to the open-ended question.

As far as software professionals' responses were concerned, the most recurring top two themes emerged to be Focus towards skills with $22 \%$ followed by Skills demonstration with 16\%. Others include Learn/Improve skills, Effective Query Resolution, Employee motivation, Holistic system, Focus on required skills, Usage of skill points, Skill points to assess skills, Upskill in-house employees, Intuitive system, Interactive learning experience, Better control to upskill, Rewards and recognition, Result-oriented and Organization access to skill pool. As far as human resource managers' responses were concerned, the most recurring top two themes emerged to be Skills demonstration with $42 \%$ followed by Focus towards skills with $33 \%$. Others include Employee motivation and Interactive learning experience.

Below are some of the participant comments highlighting the reasons why they think this system will create a skill-focused climate in the organization:

"The system you have will force employees to think in terms of skills which should automatically drive them towards upskilling. Demonstrating skills using a proof-of-concept is certainly needed rather than just theoretical knowledge. Also, utilizing gamification concepts in your system will encourage everyone to use it."

"The system is all about employee skills and how to acquire new skills. I believe knowledge sharing by demonstration will help in effective employee communication skills. The organization will have to make it a necessary system for all, only then will this system create a skill focused climate in the organization."

"It is an innovative system focused on skills. Employees will be encouraged to learn skills if rewards like badges are given. Also knowledge sharing through demonstration of skills will nurture a culture focused on skills." 


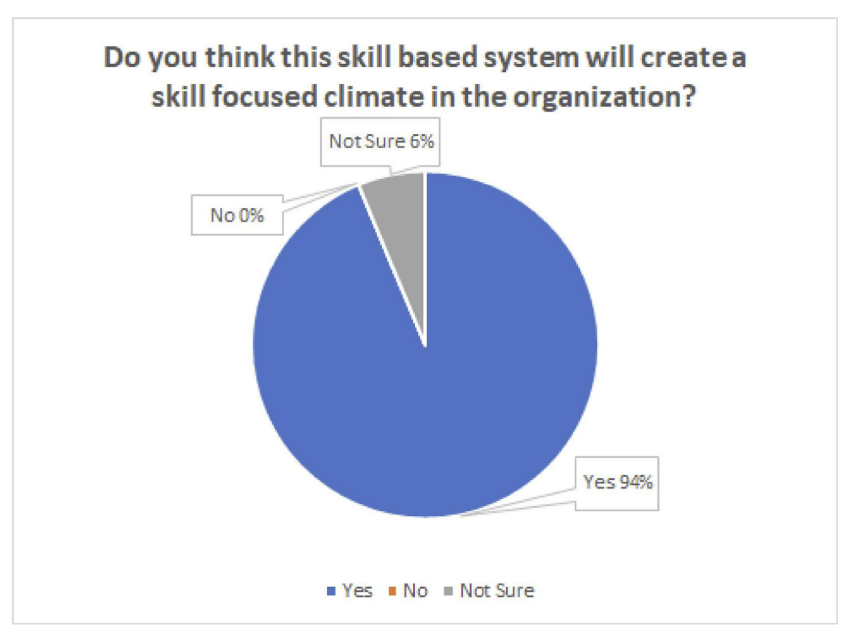

Framework of employee engaging system

(a) Software Professionals

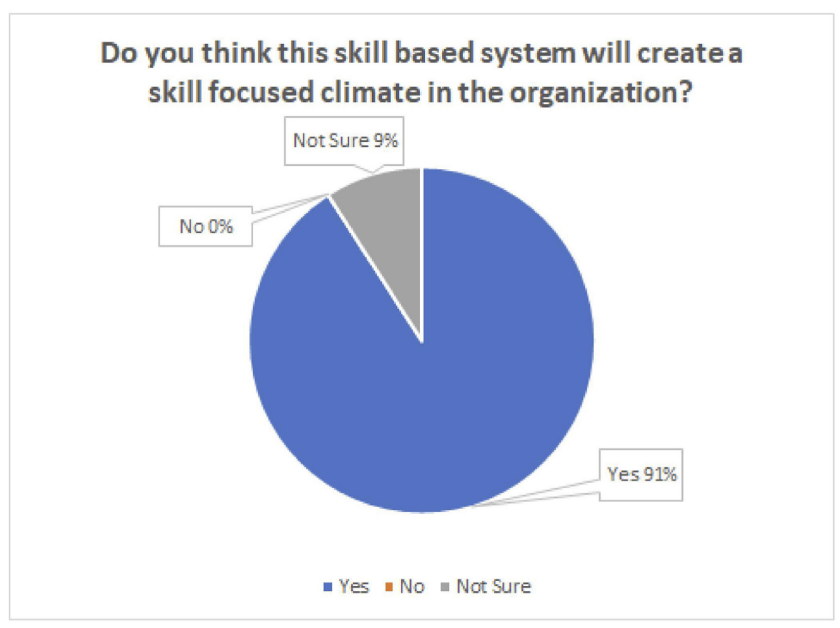

(b) Human Resource Managers

Figure 10. Ability of the skillbased system to create a skill-focused climate - Response Summary.

"Employees will focus more on sub-skills instead of just broad level skills. This focus towards sub-skills will make them more skill-focused. I liked demonstrations using proof-of concept applications - it would be inspiring to see an environment in the company where employees are demonstrating AI, Robotics and other advanced technology. It will definitely create a skill focused learning climate."

7.3 Ability of the skill-based system to utilize internal employees for new skill requirements As shown in Figure 13, over $90 \%$ of the human resource managers believe that this proposed skill-based system will help in better utilization of internal employees rather than directly hiring new external employees. The cross tabulation in Figure 14 shows the distribution of 
$\mathrm{ACI}$

$19,1 / 2$

98

98

Figure 11.

Ability of the skill-

based system to

create a skill-focused

climate - Cross

Tabulation Analysis.
Do you think this skill based system will create a skill focused climate in the organization?

\begin{tabular}{|l|l|r|r|r|r|}
\hline Frequency & Experience & & & & \\
\hline Response & $0-4$ years & 4-6 years & 6-10 years & 10+ years & Grand Total \\
\hline No & 0 & 0 & 0 & 0 & 0 \\
\hline Not sure & 0 & 1 & 2 & 0 & 3 \\
\hline Yes & 17 & 9 & 12 & 7 & 45 \\
\hline Grand Total & 17 & 10 & 14 & 7 & 48 \\
\hline
\end{tabular}

\begin{tabular}{|l|r|r|r|r|r|}
\hline Percent by Response & Experience & & & & \\
\hline Response & $0-4$ years & 4-6 years & 6-10 years & 10+ years & Grand Total \\
\hline Not sure & $0.00 \%$ & $33.33 \%$ & $66.67 \%$ & $0.00 \%$ & $100.00 \%$ \\
\hline Yes & $37.78 \%$ & $20.00 \%$ & $26.67 \%$ & $15.56 \%$ & $100.00 \%$ \\
\hline Grand Total & $35.42 \%$ & $\mathbf{2 0 . 8 3} \%$ & $\mathbf{2 9 . 1 7 \%}$ & $\mathbf{1 4 . 5 8} \%$ & $\mathbf{1 0 0 . 0 0 \%}$ \\
\hline
\end{tabular}

\begin{tabular}{|l|r|r|r|r|r|}
\hline Percent by Experience & Experience & & & & \\
\hline Response & $\mathbf{0 - 4}$ years & 4-6 years & $\mathbf{6 - 1 0}$ years & $\mathbf{1 0 +}$ years & Grand Total \\
\hline Not sure & $0.00 \%$ & $10.00 \%$ & $14.29 \%$ & $0.00 \%$ & $6.25 \%$ \\
\hline Yes & $100.00 \%$ & $90.00 \%$ & $85.71 \%$ & $100.00 \%$ & $93.75 \%$ \\
\hline Grand Total & $\mathbf{1 0 0 . 0 0 \%}$ & $\mathbf{1 0 0 . 0 0 \%}$ & $\mathbf{1 0 0 . 0 0 \%}$ & $\mathbf{1 0 0 . 0 0 \%}$ & $\mathbf{1 0 0 . 0 0 \%}$ \\
\hline
\end{tabular}

(a) Software Professionals

Do you think this skill based system will create a skill focused climate in the organization?

\begin{tabular}{|c|c|c|c|c|}
\hline Frequency & Experience & & & \\
\hline Response & $0-4$ years & 4-6 years & 6-10 years & Grand Total \\
\hline No & 0 & 0 & 0 & 0 \\
\hline \begin{tabular}{|l|} 
Not Sure \\
\end{tabular} & 0 & 0 & 1 & 1 \\
\hline Yes & 4 & 2 & 4 & 10 \\
\hline Grand Total & 4 & 2 & 5 & 11 \\
\hline
\end{tabular}

\begin{tabular}{|l|r|r|r|r|}
\hline Percent by Response & Experience & & & \\
\hline Response & 0-4 years & 4-6 years & 6-10 years & Grand Total \\
\hline Not Sure & $0.00 \%$ & $0.00 \%$ & $100.00 \%$ & $100.00 \%$ \\
\hline Yes & $40.00 \%$ & $20.00 \%$ & $40.00 \%$ & $100.00 \%$ \\
\hline Grand Total & $\mathbf{3 6 . 3 6} \%$ & $\mathbf{1 8 . 1 8} \%$ & $\mathbf{4 5 . 4 5} \%$ & $\mathbf{1 0 0 . 0 0 \%}$ \\
\hline
\end{tabular}

\begin{tabular}{|l|r|r|r|r|}
\hline Percent by Experience & Experience & & & \\
\hline Response & $0-4$ years & $4-6$ years & 6-10 years & Grand Total \\
\hline Not Sure & $0.00 \%$ & $0.00 \%$ & $20.00 \%$ & $9.09 \%$ \\
\hline Yes & $100.00 \%$ & $100.00 \%$ & $80.00 \%$ & $90.91 \%$ \\
\hline Grand Total & $100.00 \%$ & $100.00 \%$ & $100.00 \%$ & $100.00 \%$ \\
\hline
\end{tabular}

(b) Human Resource Managers 


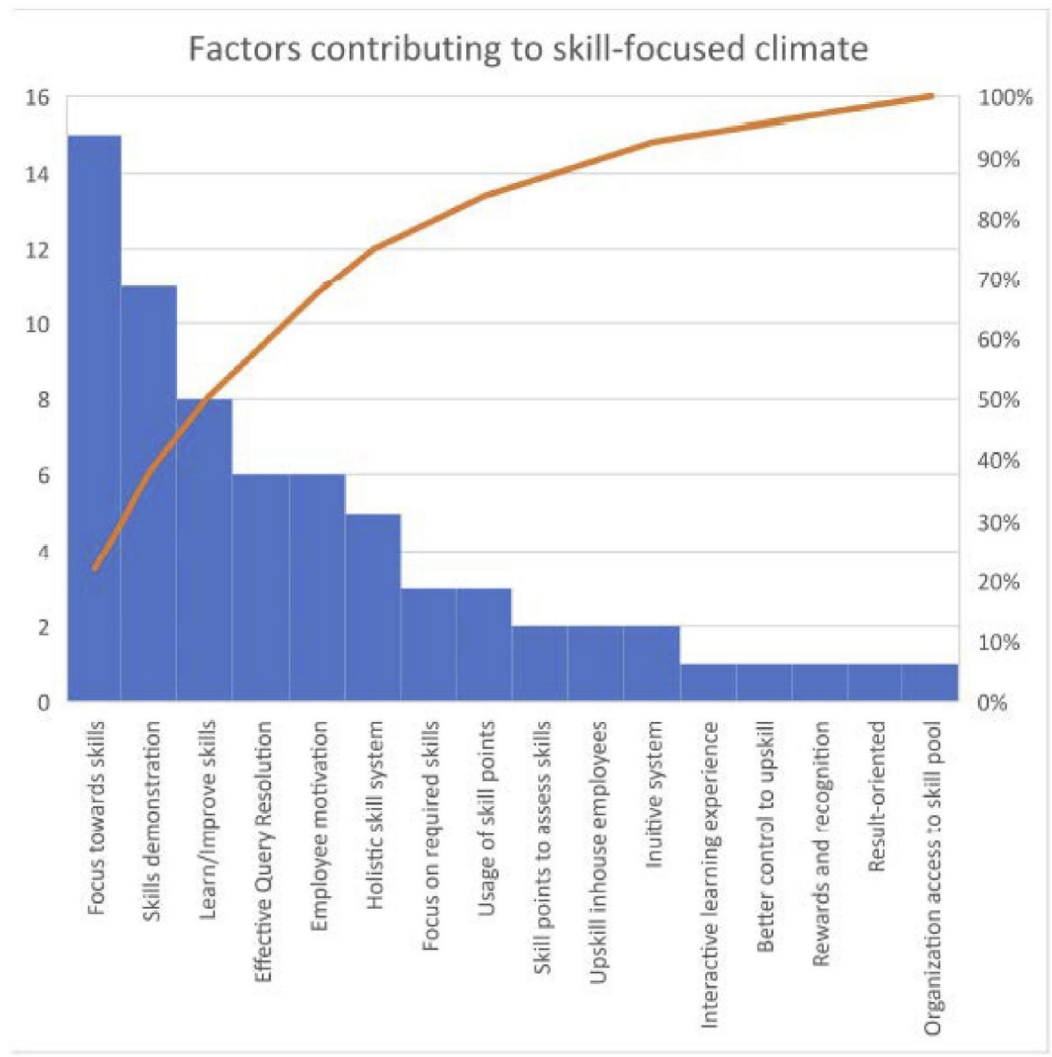

Framework of employee engaging system

99

(a) Software Professionals

\section{Factors contributing to skill-focused climate}

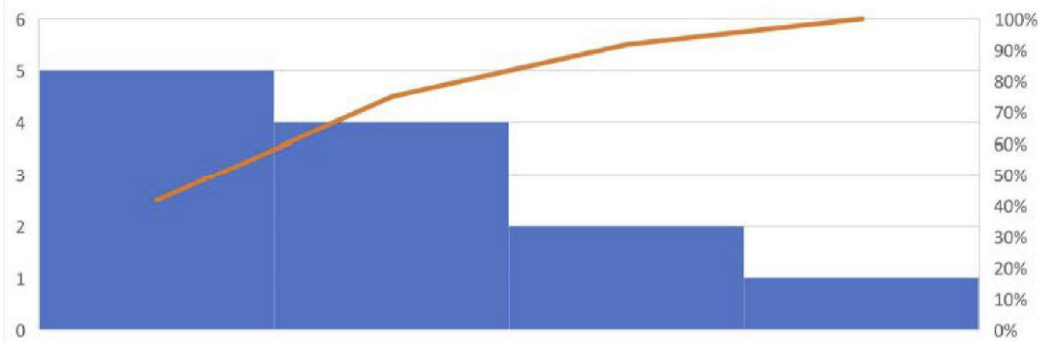

Figure 12

Thematic Analysis Pareto diagram.

(b) Human Resource Managers 


\section{$\mathrm{ACI}$ $19,1 / 2$}

$\begin{array}{ll}\text { Theme } & \text { Description } \\ \begin{array}{l}\text { Effective Query } \\ \text { Resolution }\end{array} & \begin{array}{l}\text { The system helps to reach out to the right } \\ \text { experts to resolve technical queries }\end{array}\end{array}$

Focus towards skills

Learn/Improve skills

\section{Employee motivation \\ Skills \\ demonstration}

Focus on required skills

\begin{abstract}
Skill points to assess skills
\end{abstract}

Interactive learning experience

Better control to upskill

Holistic skill system
The system is focused towards skills of

The system helps to learn new skills and improve existing skills.

The system motivates employees to upskill.

The system allows employees to demonstrate their skills using proof-ofconcepts to other employees in the organization.

The system dashboard displays the required role-level skill points that helps the employee to focus on those on priority.

The system feature of accessing each skill using skill points help to create a skill-focused climate.

The system provides an interactive learning experience.

The employees have better control of their skills.

The proposed system in its entirety with the features help to create a skill-focused climate. the employee

\section{Example}

"The employees will have someone to share their opinion with or someone to look up to for their queries thereby reducing the need of external sources."

"I can clearly see you proposing a system with skills as top priority. If a system talks about skills and if the organization enforces this system to be used by everyone, definitely I see a skill focused climate."

"This solution will give the employees an opportunity to evaluate their skills, setup their goals and enable them to interact with like minded people willing to learn and teach the specific skills one is interested in."

"Employees would be motivated to enhance their skills by learning and sharing their knowledge."

"This system will help software developers to demonstrate their skills. All developers have skills and if they demonstrate it, a learning culture will be created."

"It will be clear for all employees whatever skills are needed for each task/project and they'll be constantly reminded to work on their skills in order to reach new positions within the company what doesn't always happens."

"The points system will help everyone focus on the skills needed at a role and project level thereby increasing the focus on skills."

"It is good, very enhanced and interactive learning. It will enhance ones personal skill to maximum and thus help in organizational growth"

"Simply because the entire process of enhancing one's skill will become more reachable. So, the employees will put in efforts because they now have the access to horne their skills. In return, the organization will get a pool of skilled resources."

"This system captures all sought of skills required for an employee growth, even problem solving skills through query resolution. So a skill focused climate will be created."
Table 2.

Thematic Analysis Common Themes. 


\begin{tabular}{ll}
\hline Theme & Description \\
\hline $\begin{array}{l}\text { Upskill inhouse } \\
\text { employees }\end{array}$ & $\begin{array}{l}\text { The system can be used to upskill } \\
\text { internal employees in case of skills } \\
\text { deficit. }\end{array}$
\end{tabular}

Rewards and recognition

Intuitive system

Result-oriented

Organization access to skill pool
Employee recognition by giving badges will create an interest to learn and improve.

The system is easy to use and understand.

The system aims towards upskilling employees.

The system helps in providing the organization better access to all the available skills.

\section{Example}

"It is important for an employee to aquire new skills and share the skills that he/she is expert in. This will lead to lesser attrition of employees seeking growth and learning opportunities and also beneficial for an employer where he need not hire external resource if he has the required skillset within."

"Rewards and recognition, demonstration of skills to others will spark enthusiasm and interest to learn, improve and be proactive."

"Its intuitive and helps cultivate learning culture."

"Framework is more focused and result oriented towards upskilling their employees and in longer run it will be beneficial to organization."

". . . the organization will be aware of all skills present and will hence be able to appropriately utilize all the skills without spending time and effort in getting new employees with the requisite skills..."

\section{Framework of employee engaging system}

Table 2.

participant responses with respect to their experience. Overall, around $45 \%$ of the respondents fall in the experience bracket of 6-10 years, around $36 \%$ are within $0-4$ years and the remaining $18 \%$ are from 4 to 6 years. Out of the total respondents who answered 'Yes', 40\% each comes from the human resource managers who have 6-10 years and 0-4 years of experience and the remaining $20 \%$ fall within $4-6$ years of experience. Out of the respondents who fall within the experience bracket of 6-10 years, $80 \%$ of them have answered 'Yes' and the remaining 20\% have answered 'Not Sure'. All the respondents who

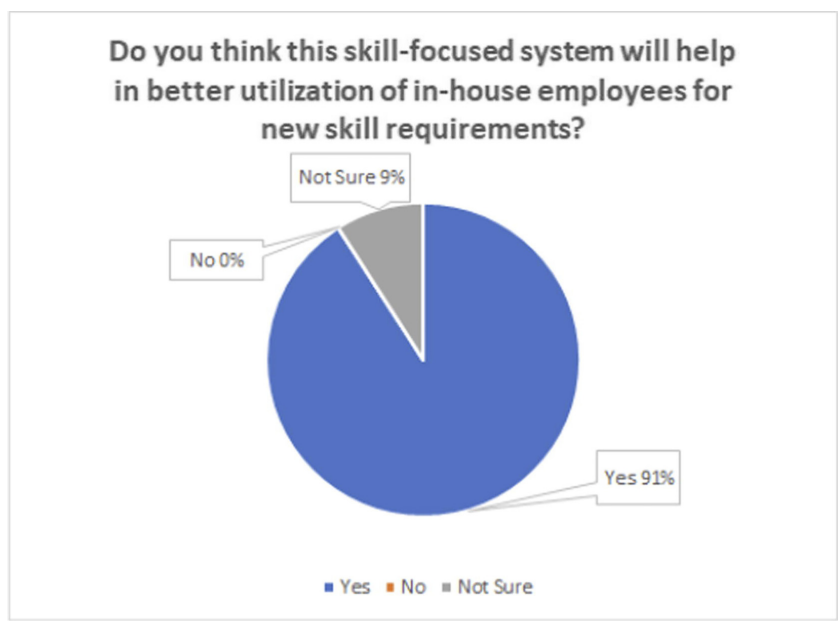

Figure 13.

Ability of the skillbased system to utilize internal employees for new skill requirements - Response Summary. 
$\mathrm{ACI}$

$19,1 / 2$

\section{2}

Figure 14.

Ability of the skillbased system to utilize internal employees for new skill requirements - Cross Tabulation Analysis.

\section{Do you think this skill-focused system will help in better utilization of in- house employees for new skill requirements?}

\begin{tabular}{|c|c|c|c|c|}
\hline Frequency & \begin{tabular}{|l|} 
Experience \\
\end{tabular} & & & \\
\hline Response & $0-4$ years & 4-6 years & 6-10 years & Grand Total \\
\hline No & 0 & 0 & 0 & 0 \\
\hline Not Sure & 0 & 0 & 1 & 1 \\
\hline Yes & 4 & 2 & 4 & 10 \\
\hline Grand Total & 4 & 2 & 5 & 11 \\
\hline
\end{tabular}

\begin{tabular}{|l|r|r|r|r|}
\hline Percent by Response & Experience & & & \\
\hline Response & $\mathbf{0 - 4}$ years & $\mathbf{4 - 6}$ years & $\mathbf{6 - 1 0}$ years & Grand Total \\
\hline Not Sure & $0.00 \%$ & $0.00 \%$ & $100.00 \%$ & $100.00 \%$ \\
\hline Yes & $40.00 \%$ & $20.00 \%$ & $40.00 \%$ & $100.00 \%$ \\
\hline Grand Total & $\mathbf{3 6 . 3 6} \%$ & $\mathbf{1 8 . 1 8} \%$ & $\mathbf{4 5 . 4 5 \%}$ & $\mathbf{1 0 0 . 0 0 \%}$ \\
\hline
\end{tabular}

\begin{tabular}{|l|l|r|r|r|}
\hline Percent by Experience & Experience & & & \\
\hline Response & 0-4 years & 4-6 years & $\mathbf{6 - 1 0}$ years & Grand Total \\
\hline Not Sure & $0.00 \%$ & $0.00 \%$ & $20.00 \%$ & $9.09 \%$ \\
\hline Yes & $100.00 \%$ & $100.00 \%$ & $80.00 \%$ & $90.91 \%$ \\
\hline Grand Total & $\mathbf{1 0 0 . 0 0 \%}$ & $\mathbf{1 0 0 . 0 0 \%}$ & $\mathbf{1 0 0 . 0 0 \%}$ & $\mathbf{1 0 0 . 0 0 \%}$ \\
\hline
\end{tabular}

are in $0-4$ and $4-6$ years of experience tend to believe this system will help in better utilization of in-house employees for new skill requirements.

Below are some of the actual responses answered by the human resource managers:

"[This skill-based system] will allow employers to staff projects with existing resources. [This skill-based system] Gives advancement and engagement opportunities to staff. [This skill-based system] Identifies skills deficits for staff who have expressed interest in career progression into a certain role and allows time for up-skilling to bridge those gaps. [This skill-based system] Saves the organization money and time in recruitment of external hires. [This skill-based system] Saves money by potentially removing the need for third party vendors/consultants/agencies. The organization has a comprehensive database of the skills of it's employees. [This skill-based system] Show's the company as an employer who is pro-actively interested in retaining and advancing existing staff by offering training and growth opportunities."

"Employees in bench can keep a watch for upcoming projects and upskill to meet the project requirements. Again this can be tough for urgent project requirements where time is constrained. But, this system has the potential in better utilization of in-house employees for sure."

"This system can be used to bring bench employees to attention for new projects. If they learn and demonstrate the required skill, the project managers can take a call to allocate them to their projects rather than directly hiring from outside."

7.3.1 Participant comments/suggestions. Below are some of the suggestions and comments received by the software professionals about this skill-based system:

"Idea looks good and promising. Future scope - Learning progress of an employee can be tracked. This data can also be used as input to annual goal setting and appraisal system of an organization."

"This approach is very well thought out and will actually help the organization achieve it's goals and promote a skill worthy environment." 
"Use data mining technologies to derive observations on entered skills."

"A must have system for software organizations."

"You must try this practically in a company and study to make this a reality. Software developers should love such atmosphere of knowledge demonstrations."

"It looks to me like a complete system that will help employee to grow professionally as well as socially due to work-life balance features."

"Good system for software developers."

Below are some of the comments and suggestions given by the Human resource managers:

"You need to bring this system to practice in a large software organization and evaluate. Your system in truly innovative and interesting..."

"I know this is just a proof-of-concept skill-based system but if you build it for large enterprise organizations, add in online learning, employee can teach skills online so that interested employees can learn from their desk."

"Bring this idea to reality, if possible. I liked your thinking. You can add data analytics to mine skills from entered skill pool to generate skill insights."

"Can be extended to add new features like technical blogs and discussion forums."

"Lots of potential in large software organizations."

\section{Discussion}

\subsection{General perspectives}

Majority of the responses received are from software professionals and human resource managers who are working in a large software organization. Also, majority of the software professional respondents are in the bracket of 0-4 years of experience. On the human resource managers side, majority of the respondents were from 6-10 years experience range. The findings after data analysis reveal the following:

1. There are large organizations that doesn't follow skill-based systems to encourage employees to learn and share knowledge.

2. Not all organizations focus on knowledge sharing by demonstration of skills to upskill their employees.

3. Almost half of the participants of the survey are not aware of the technical experts of various domains in their organization and majority of them are from large organizations.

4. Majority of the human resource managers said they directly recruit new employees with the required skill set rather than upskilling existing employees.

5. There are software professionals who are not aware of the required skills for their role.

\subsection{System feedback}

The participants liked all the below four main features of the skill-based system, but both the software professionals as well as human resource managers liked the feature of demonstrating skills more than the others.

1. Drill down questions extracting the sub skills apart from the skills

2. Knowledge sharing through demonstration of skills

3. Use of skill points to assess each skill

4. Role of skill experts in this skill-based system

Framework of employee engaging system 
$\mathrm{ACI}$

$19,1 / 2$

\section{4}

Majority of the survey participants tend to agree to each of the below points:

1. This skill-based system will make the employees more focused on their skills.

2. This skill-based system is more reliable for query resolution as it helps the employees to reach out to the right experts to resolve technical queries.

3. This skill-based system will create an encouraging learning environment in the organization to learn new skills.

4. This skill-based system will create a knowledge sharing environment in the organization.

5. This skill-based system will be beneficial to the employees in enhancing their skills.

6. This skill-based system will be beneficial to the organization in creating skilled employees.

The participant comments reveal that demonstration of skills using proof-of-concepts will help to learn emerging trends like artificial intelligence and other advanced technologies.

\subsection{Answering the research questions}

An overwhelming majority of software professionals as well as human resource managers have agreed that this proposed skill-based system will help to create a skill-focused climate in the organization. The system's focus towards skills and demonstration of skills has emerged as top two reasons in creating a skill-focused climate in the software organization. The system's focus towards skills includes the role of sub-skills considered in the system apart from the high level skills. The skills demonstration theme includes knowledge sharing through demonstration of skills using a proof-of-concept application. Also, majority of the human resource managers tend to agree that this proposed skill-based system will help in utilizing in-house employees for new opportunities rather than hiring external employees.

\subsection{Limitations of the research}

Firstly, the proposed skill-based system is an enterprise wide application suited for software employees. Respecting the time constraints, the researcher tried to fit in minimum viable features in the skill-based system. In a real-world scenario, a skill-based system can encompass a huge volume of skills and features. Also, the survey received only $37 \%$ response rate from Human resource managers. If more human resource managers had participated in the survey, it may have brought in new perspectives, thereby adding more value to the research.

\section{Conclusion \& future work}

This research has shown that a skill-focused climate can be created in an organization using a software system. The proposed skill-based system will help a software organization to create a skill-focused climate. The system's ability to make the employees focused towards their skills and an ability to help them demonstrate their skills are the two key factors that makes the system skill-oriented. This skill-based system will be highly beneficial not only to the employees but also the organization as a whole improving employee engagement. This skillbased system will encourage employees to learn and share their knowledge by demonstrating their skills leading to innovation in the organization. This skill-based system will also help in effective query resolution by reaching out to right technically sound employees to resolve their technical queries. Also, this system will help in better utilization of their in-house 
employees for new opportunities instead of hiring external candidates. This skill-based system will become a driving force for both the employees as well as the organization towards upskilling and more importantly will lead to a change in perspective towards skills thereby creating a skill-focused learning climate within the software organization.

The suggestions given by the software professionals and the human resource managers can be implemented to the skill-based system. Technologies like data analytics and data mining can be introduced into the system to derive interesting information on employee skills and their learning progress trends. Also, apart from the minimal viable features implemented in the skill-based system the rest of the features can be implemented along with the aforementioned suggestions.

\section{Notes}

1 https://www.google.com/forms/about/.

2 https://www.youtube.com/watch?v=Lps_VeNJIy4.

\section{References}

[1] L. Eldor, I. Harpaz, Retracted: The indirect relationship between learning climate and employees' creativity and adaptivity: The role of employee engagement, Pers. Psychol. 69 (3) (2016) E1-E44.

[2] R. Bates, S. Khasawneh, Organizational learning culture, learning transfer climate and perceived innovation in jordanian organizations, Int. J. Training Devel. 9 (2) (2005) 96-109.

[3] L. Eldor, I. Harpaz, A process model of employee engagement: the learning climate and its relationship with extra-role performance behaviors, J. Organizational Behav. 37 (2) (2016) 213-235.

[4] A.I. Ismail, Employee learning theories and their organizational applications, Acad. J. Econ. Stud. 3 (4) (2017) 96-104.

[5] A. Kaushik, S. Xu, Derivation of customer intelligence from customer knowledge management, Intelligence (CI) 2728.

[6] S. Naithani, A. Kaushik, Evaluation and comparison of the conceptual frameworks of cms, Int. J. Comput. Sci. Eng. (IJCSE) 8 (8) (2016) 357-361.

[7] M. Ozer, D. Vogel, Contextualized relationship between knowledge sharing and performance in software development, J. Manage. Inform. Syst. 32 (2) (2015) 134-161.

[8] H.-W. Lee, Demonstrating the importance of interactional socialization in organization, Syst. Res. Behav. Sci. 28 (3) (2011) 264-269.

[9] K. Malik, Impacts of organizational knowledge sharing practices on employees' job satisfaction: Mediating roles of learning commitment and interpersonal adaptability, J. Workplace Learn. 30 (1) (2018) 2-17.

[10] A. Felstead, D. Gallie, F. Green, Y. Zhou, Employee involvement, the quality of training and the learning environment: an individual level analysis, Int. J. Human Resource Manage. 21 (10) (2010) $1667-1688$.

[11] C. Lejeune, D. Mercuri, S. Beausaert, I. Raemdonck, Personal development plans supporting employee learning and perceived performance: the moderating role of self-directedness, Human Resource Devel. Int. 19 (4) (2016) 307-328.

[12] S. Deterding, D. Dixon, R. Khaled, L. Nacke, From game design elements to gamefulness: defining gamification, in: Proceedings of the 15th International Academic MindTrek Conference: Envisioning Future Media Environments, MindTrek '11, ACM, New York, NY, USA, 2011, pp. 9-15, https://doi.org/10.1145/2181037.2181040.

[13] A.M. DuVernet, E. Popp, Gamification of workplace practices, Ind.- Organizational Psychol. 52 (1) (2014) 39-44. 
$\mathrm{ACI}$

$19,1 / 2$
[14] J. Münch, F. Fagerholm, P. Johnson, J. Pirttilahti, J. Torkkel, J. Jäarvinen, Creating minimum viable products in industry-academia collaborations, in: Lean Enterprise Software and Systems, Springer, 2013, pp. 137-151.

[15] I. Sommerville, Software Engineering, 10th Edition., Pearson, 2015.

[16] M.N. Saunders, Research methods for business students, 7/e, Pearson Education, India, 2015.

\section{6}

\section{Appendix A}

\section{Skills Dashboard Find Expert Give Query Badge Track Requests}

Siddarth Nair

Search

\section{Dashboard}
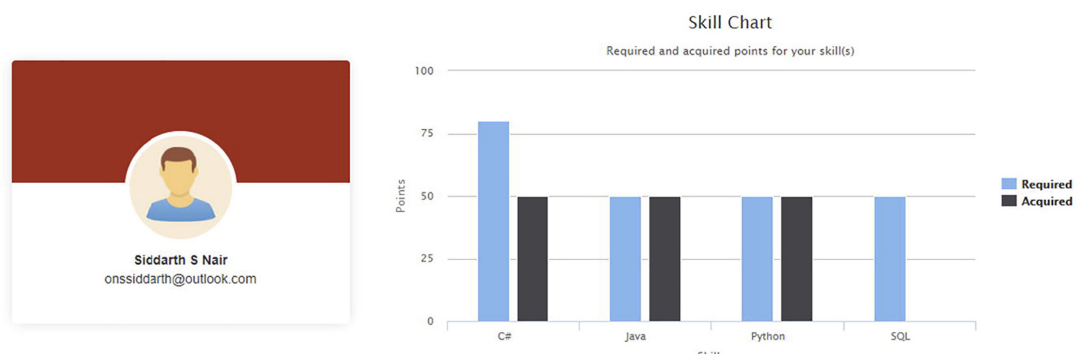

Figure 15.

View other's dashboard.

Figure 16.

Employee

Demonstration

Request screen.

\section{Skills Dashboard Find Expert Give Query Badge Track Requests}

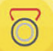

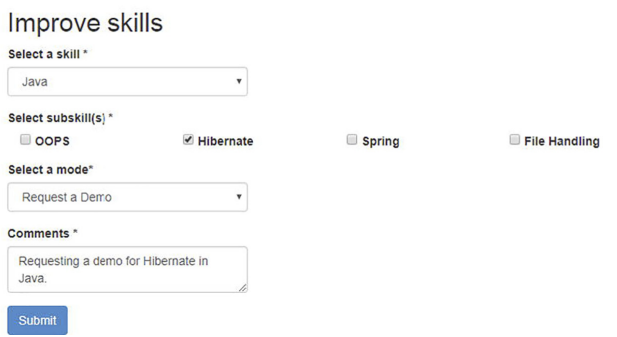




\section{Skills Dashboard Find Expert Give Query Badge Track Requests}

\section{Give Query Badge}

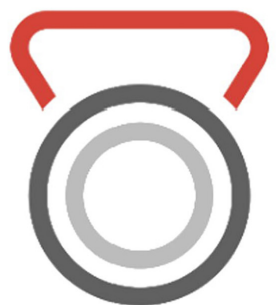

Select badge recepient *

Siddarth Nair

Comments *

Thanks for helping me with $\mathrm{C}$ \#

Inheritance issue.
Framework of employee engaging system

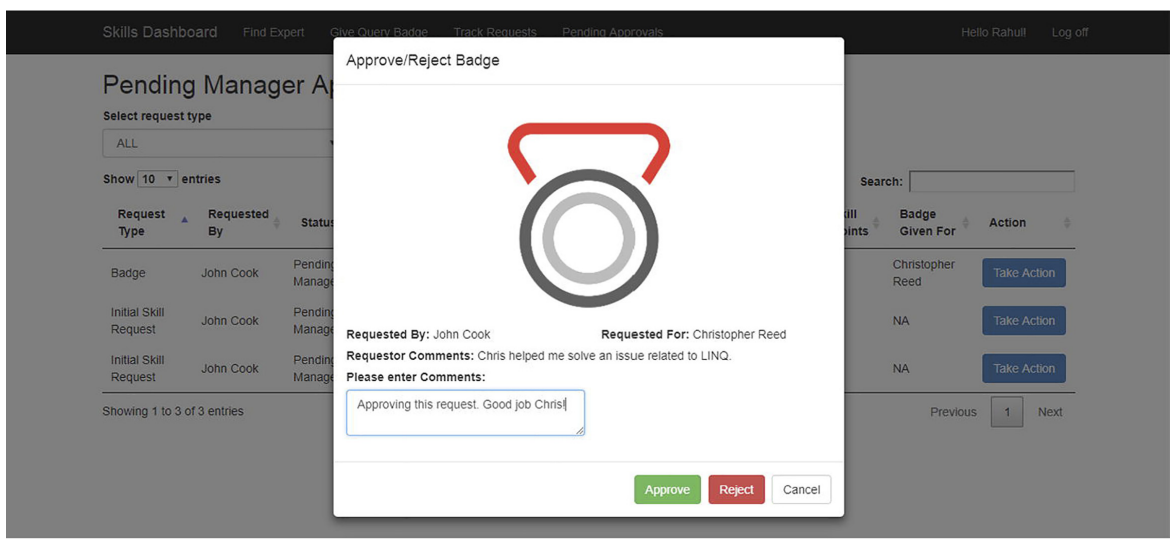

Figure 17.

Employee gives a query badge.

Figure 18. Manager Badge Approval screen.

\section{Corresponding author}

Siddarth Nair can be contacted at: 10376001@mydbs.ie

For instructions on how to order reprints of this article, please visit our website:

www.emeraldgrouppublishing.com/licensing/reprints.htm

Or contact us for further details: permissions@emeraldinsight.com 